\title{
REVIEW
}

\section{Pelvic floor and sexual male dysfunction}

\author{
Antonella Pischedda ${ }^{1}$, Ferdinando Fusco ${ }^{2}$, Andrea Curreli ${ }^{1}$, Giovanni Grimaldi ${ }^{2}$, \\ Furio Pirozzi Farina ${ }^{1}$ \\ ${ }^{1}$ Azienda Ospedaliera Universitaria di Sassari, Italy; \\ ${ }^{2}$ Azienda Ospedaliera Universitaria Federico II di Napoli, Italy.
}

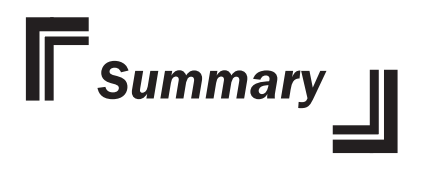

The pelvic floor is a complex multifunctional structure that corresponds to the genito-urinary-anal area and consists of muscle and connective tissue. It supports the urinary, fecal, sexual and reproductive functions and pelvic statics.

The symptoms caused by pelvic floor dysfunction often affect the quality of life of those who are afflicted, worsening significantly more aspects of daily life. In fact, in addition to providing support to the pelvic organs, the deep floor muscles support urinary continence and intestinal emptying whereas the superficial floor muscles are involved in the mechanism of erection and ejaculation. So, conditions of muscle hypotonia or hypertonicity may affect the efficiency of the pelvic floor, altering both the functionality of the deep and superficial floor muscles. In this evolution of knowledge it is possible imagine how the rehabilitation techniques of pelvic floor muscles, if altered and able to support a voiding or evacuative or sexual dysfunction, may have a role in improving the health and the quality of life.

KEY WORDS: Pelvic floor; Physical therapy; Sexual dysfunction; Pelvic floor dysfunction.

\section{INTRODUCTION}

The pelvic floor is a complex multifunctional structure made of both muscular and tendineus components. In fact, in addition to providing support to the pelvic organs, the pelvic floor muscles active the peripheral mechanisms of urinary and fecal continence and the evacuation facilitators (1). So, pelvic floor has an important role in the control of bowel and bladder functions. Besides this, recently it is emerging the importance of pelvic floor muscles (PFM) on male and female sexual function. In the male, in particular, have been highlighted the ways in which contractions/relaxation of PFM are involved in the mechanisms of erection (2) and ejaculation (3). On this basis, it was postulated that some changes in erectile and ejaculatory function may be secondary to anatomical and functional perineal muscles alterations. Finally, and most recently, it has been shown that some algic phenomena evoking symptoms due to an inflammatory prostato-vesicular process, can be supported by functional alteration of the PFM (4).

The symptoms of dysfunction of pelvic floor muscles were divided into 5 groups by ICS (Table 1). In this evolution of knowledge it is possible to imagine how the rehabilitation techniques of PFM, if altered and able to support a voiding or evacuative or sexual dysfunction, may have a role in improving the health and the quality of life (6).

Nevertheless, it would seem that the approach to pelvic floor disease related dysfunction is still performed only from a restricted medical group and stenting to become well established in urologists' routine clinical practice. This article will get closer to the uro-andrologist intriguing possibilities offered by a better understanding of the correlation between pelvic floor dysfunction and male sexual dysfunction.

\section{ANATOMY AND PHYSIOLOGY OF THE PELVIC MUSCLES OF MALE}

The differences between male and female urogenital anatomy, also mark some anatomical and functional dif- 
Table 1.

Symptoms of pelvic floor muscles dysfunction.

1.1. Low urinary tract syntoms
· urinary incontinence
· frequency/urgency
· poor or intermittent stream initiated or supported
by muscular effort
- hesitancy
- terminal dribbling
- incomplete voiding

\subsection{Bowel syntoms}

- obstructed defecation

- constipation

- fecal incontinence

- rectal/anal prolapse

\subsection{Vaginal syntoms}

- pelvic organ prolapse

\subsection{Sexual function}

- in women: dyspareunia

- in the male: erectile dysfunction and ejaculatory disorders

- both: orgasmic dysfunction

\subsection{Pain}

- pelvic pain

- cronic pelvic pain syndrome (cpps) ferences of the muscle-tendon complex that forms the pelvic floor. That said, in both sexes the pelvic floor muscles are equal and symmetrical and form the pelvic diaphragm that covers the pelvic cavity from the front portion to the rear. The diaphragm supports the bladder and the rectum and is on two levels: the deep and superficial one (1) (Figures 1-2).

The deep muscles of the plan can be represented in two layers: the outer layer, represented by pubo-coccygeal muscle, ileum-coccygeal and ischium-coccygeal, the inner layer is represented by the pubo-rectalis muscle. The muscles pubo-coccygeal (PC), pubo-rectalis and ischio-coccygeal form a functional unit known as the levator ani muscle (LAM). The pelvic diaphragm is completed, posteriorly, by ilio-coccygeal and ischio-coccygeal muscles. The superficial floor musculature is made up of the bulbo-cavernous, ischio-cavernous, external sphincter and superficial transverse perineal muscle (Figure 3).

This muscle floor plays an important role in erectile function, ejaculation and in the anal sphincter mechanism.

The activity of the PC muscle expresses the overall functionality of the LAM which is a good measure of definition of the pelvic floor muscles activity as a functional whole. For this reason, through the PC functional study, it is possible implement a reliable diagnostic and therapeutic approach to disorders of the PFM.

The PFM is constituted, for approximately two thirds, by

Figure 1.

Pelvic diaphragm of male - inferior view.

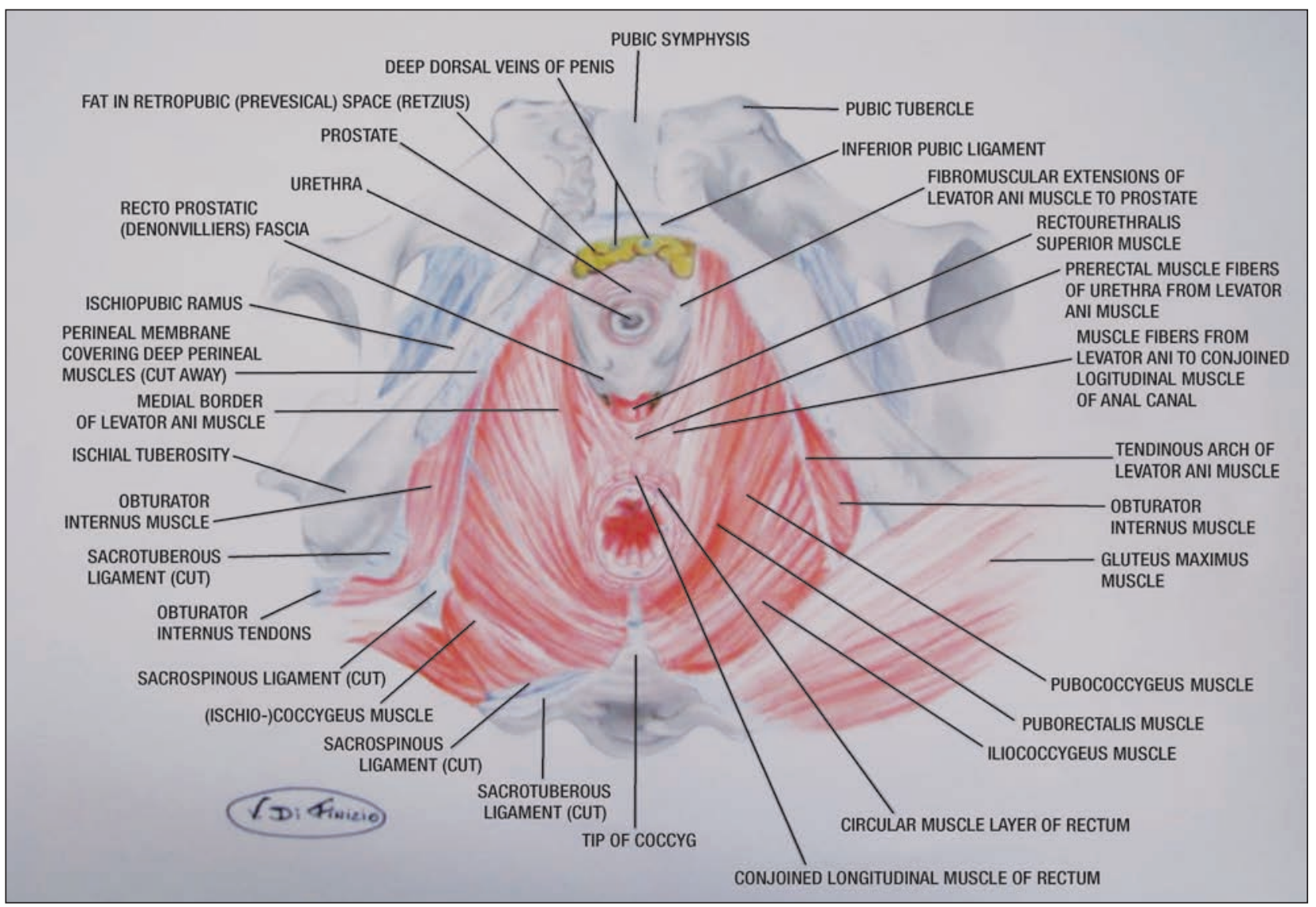


Figure 2.

Pelvic diaphragm of male - inferior view - viscera removed.

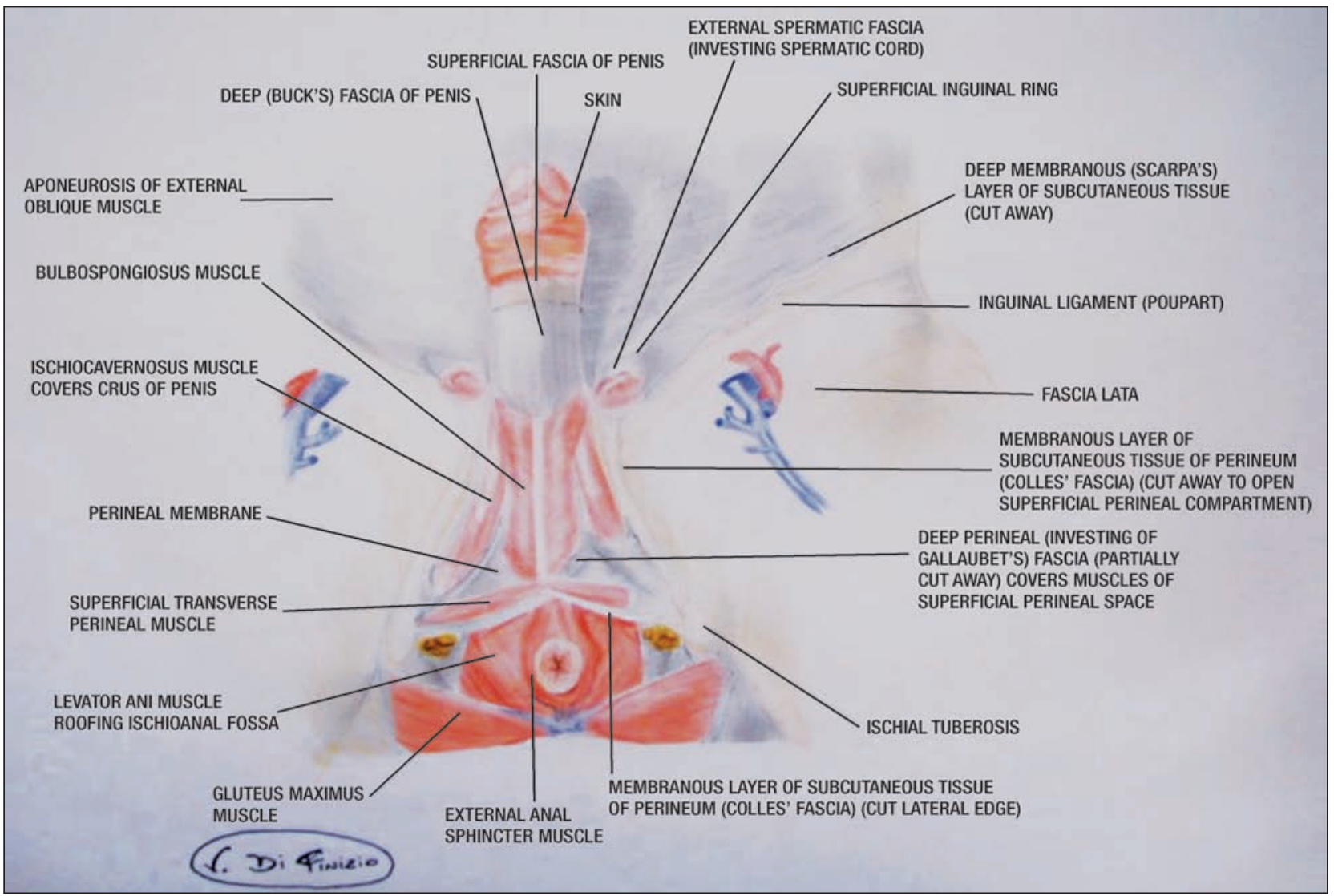

Figure 3

Perineum and external genitalia of male - deep dissection.




slow twitch fibers (type 1) and for about one third of fast-twitch fibers (type 2) (7). Slow twitch fibers are stably in a tonic contraction and this muscle tone allows the PFM to support the pelvic organs (7). The sphincteric muscles, including the periurethral, consists of fibers of type 1 and 2 since it must fulfill a dual function: increasing the urethral endurance during the period of raised intra-abdominal pressure (fiber type 1) and the voluntary control of sphincters (fiber type 2) (8).

\section{SCHEMES OF USE OF THE PELVIC FLOOR MUSCLES}

PFM usage patterns are acquired in childhood and scheduled in cerebral mechanism. Afferent stimuli generated by the voiding and sexual apparatus determine a central processing aimed the most appropriate response model of the PFM (10). In this way, in the course of its growth, people gradually learn to "hold" urination, feces and intestinal gas, but also to modulate the relaxation and contraction of the pelvic muscles to handle in the best way the ejaculation. These capabilities are in part related to adequate corticalization of the PFM and, overall, to the efficiency of muscle tone. So, conditions of muscle hypotonia or hypertonicity may affect the efficiency of the pelvic floor, altering both the functionality of the deep and superficial floor muscles. While the alterations of the deep floor muscles may determine effects on urinary continence and intestinal emptying, the alterations of the superficial floor muscles may affect the quality of the erection and ejaculation. In fact it is only under conditions of optimal tonicity that these muscles perform compressive action necessary to avoid the outflow of blood from the crura (m.ischio-cavernous) (1) and the periurethral glans along the spongiosa (m. bulbo-cavernous). With regard to erectile function, Grace Dorey (2000) conducted a review of the literature showed that the efficiency of the PFM is higher in powerful men than those affected by erectile dysfunction (ED) (11).

\section{The hypertonicity of the pelvic floor muscles}

In cases in which the PFM is chronically incorrectly used, for example when the subject takes the habit of delaying urination or defecation voluntarily by contracting the pelvic musculature, you can establish special patterns chronic pelvic floor summarized under the term "hypertonic" (1).

Other dysfunctional patterns are those resulting from unsuitable activation in voiding phase of perineal muscles. In these cases, the muscle groups most frequently involved are the abdominal muscles, the diaphragm, the adductors and gluteal muscles (12). When this happens, frequently are established perineal muscular synergies that can be of either agonist or antagonist kind (13). In the agonists synergies, in conjunction with the levator ani contraction, it determines the activation of the adductor muscles and buttocks; in antagonistic synergies, levator ani simultaneously activates the abdominal muscles and the diaphragm (13). The causes of dyssynergia can be congenital or acquired (10). As a congenital cause, has been suggested a poor perineal region corticalization (10). Among the acquired causes, first of all, behavioral causes. Then, reduced pelvic floor muscles efficien- cy and the psychological cause (10). In case of activation of the abdominal press, it is established a hypertonic reflection of the PFM that expresses a condition of antagonist muscolar synergism (4). Over the time, incongruous patterns of emptying and a state of chronic contraction of the PFM will also involve the anorectal system and ischiocavernous and bulbo-cavernous muscles (4).

In more severe cases, the antagonist synergy may increase up to become a dyssynergia. This happens when patient, rather than activate the levator ani muscle, only contracts the abdominal muscles, taking a particular pattern called "inversion of perineal control" (12).

In addition, since superficial floor muscles form a functional whole with deep floor ones, the hypertonicity of the PFM and the dyssynergia may come to represent a correlation factor between functional urological alterations and some types of sexual dysfunction (1). In this way it can be caused, or aggravate, premature ejaculation. As regards erectile function, ischio-cavernosa muscle hypertonicity, however it is to determine, may support a reduction of volume expansion capacity of the roots of the corpora cavernosa with consequent reduction of the maximum rigidity.

\section{The hypotonia of the muscles of the pelvic floor}

The condition of hypotonia of the PFM is less frequent in men than in women, in which pregnancy and vaginal delivery (especially in multiparous), obesity, aging and, more discussed, menopausal characteristic hormonal changes (14) are also risk factors of dysfunction (15).

Although the condition of the PFM hypotonia may have a neurogenic, (1) malformative (16) and iatrogenic postsurgical cause (1). In the male tissue aging-associated changes are the most common cause of muscular hypotonia. This assertion is supported by the finding that the efficiency of the PFM decreases with aging and aging self correlates with an increase of ED (11). This is because with aging, the number of muscle fibers reduces and collagen undergoes both qualitative and quantitative changes, involving muscle bulbo- and ischio-cavernous hypotonia, and consequently changes in the cavernous veno-occlusive mechanism and post-void dribbling. (17) In support of this, Strasser et al. (1997), in a study on human rabdomiosphincter, found a positive correlation between age progression and quantitative decrease of striated muscle fibers so that, in this muscle, fibers are represented as follows: $79 \%$ in childhood and $35 \%$ at 85 years (18).

Finally, although in the literature there is still not accordance, it would appear that both the PFM hypotonus and the hypertonicity can support a premature ejaculation, in these cases secondary to reduced efficiency of perineal contraction/decontraction mechanisms having a role in the control of the ejaculatory reflex (1).

\section{The Clinical eVAluation OF PELVIC FLOOR}

The PFM correct functionality is linked to its normal morphological development and the integrity of its neuro-vascular component. Subject to these assumptions, muscle assessment must define the tone, strength, endurance and fatigue of the PFM. After verifying the 
absence of dysfunctional pattern of activation of the PFM, these assessments can be performed manually, introducing a flexed finger hooked in the anal canal or by kinesiological electromyography (EMG). With these methods it is possible to detect the fasic strength and various tonic expressions of the strength of the pubo-coccygeal muscle (PC test).

EMG offers the advantage of measuring the actual muscle activity in microvolts, although the high cost of the equipment makes the technique not common (1). It should be noted, however, that the instrument by which is performed the kynesiological EMG is advantageously used also as electromyographic biofeedback, in order to implement a physical therapy aimed at helping patients to optimize their muscles activity (20).

The digital rectal evaluation is used to assess the symmetry and balance of muscles that can be explored with this method, differentiating muscle able to close (puborectalis and external anal sphincter) and muscles able to lift (pubococcygeus and ilealcoccygeus) (1). In the male, intra-anal palpation is therefore considered an appropriate sensitive method to assess the strength and tone of the pelvic floor muscles (1).

\section{Physical theRAPY OF SEXUAL DYSFUNCTION SECONDARY TO THE PELVIC FLOOR MUSCLE DYSFUNCTION}

Physical therapy of sexual dysfunction secondary to pelvic floor alteration, still lacks of unique and validated benchmark. However, physiotherapeutic treatment will have to be modulated, qualitatively and quantitatively, as a function of the objectives that were set in agreement with the patient and the results obtained progressively.

Academically, physical therapy is divided in two stages: the educational and rehabilitative in the strict sense.

The educational stage is a preparation for the rehabilitation phase. It must provide, in a clear and simple information about the anatomy and physiology of the pelvic floor.

The rehabilitation phase has the aim of improving the dysfunctional phenomenology reported by the patient. It is structured according to a treatment plan tailored to suit the type of detected pelvic dysfunction.

The main objectives of physical therapy are raise awareness and proprioception of the muscles; discrimination and improve muscle relaxation; normalize muscle tone (25). To pursue these objectives, physiotherapy may use tools such as the electromyographic biofeedback, functional electrical stimulation and pelvic-perineal physiotherapy (25). Of all the physiotherapy treatments, biofeedback is only reported in controlled studies (25).

\section{Electromyographic Biofeedback}

The electromyographic biofeedback is an equipment capable of providing information on the activity of a muscle district, so the patient can re-act and change it (12).

Takes place via a rectal EMG probe associated with surface electrodes. Using digital electronic interface connected to a computer, a video with a simplified graphic expresses, real time, electromyographic activity detected (12). In this way the patient highlights, and is able to influence muscular activity escaped from his cortical control mechanisms, or knowingly to put to work the muscle under examination in order to improve the phase and/or the endurance, implementing a kind of "competition" with the machine (12).

\section{Functional electrical stimulation (FES)}

The electrical stimulation can be applied directly to the muscle or indirectly, through the stimulation of the n.pudendal fibers (12). Usually is performed using indirect stimulation that through the n.peripheral depolarisation generates a nerve impulse that causes muscle contraction (12). Administered therapeutically, these contractions help to increase muscle strength using the same mechanisms of exercise therapy. These result in two effects: the facilitation of voluntary movement of the treated muscle group and the improvement of neuromotor control (12). For these reasons, we talk about functional electrical stimulation (FES) for rehabilitation (12). Contraindications to the SEF are: the perineal complete denervation, the VU reflux, the cardiac pacemaker and urinary infections (12). It is used a biphasic current, 10 to $50 \mathrm{~Hz}$. $\cong 50 \mathrm{~mA} \times 0.2 \mathrm{msec}$.

\section{Pelvic-perineal physiotherapy \\ (Pelvic Floor Muscle Training or PFMT)}

The PFMT is the first therapeutic approach to be implemented in the home, after the patient has performed an adequate training learning (26). A good treatment schedule is applyng 1 hour treatment starting from 2 times per week and up to 4 months, then customizing the sessions in function of the results obtained progressively (27).

\section{Pelvic floor Rehabilitation therapy IN MALE SEXUAL DYSFUNCTION}

\section{Erectile Dysfunction}

The pelvic floor muscle is involved in the increased intra-cavernous pressure. In fact, as reported above, the activity of the ischiocavernosus muscle facilitates erection while the contraction of the bulbocavernosus muscle slows the venous drainage from the corpora cavernosa, exerting pressure on the deep dorsal vein of the penis (1). This implies that, in case of hypotonia of the PFM can be established erectile dysfunction with venoocclusive mechanism. This can benefit significantly from the physical rehabilitation therapy of the pelvic floor, which aims to increase strength and muscle tone and improve the fatiguability $(17,28)$. By contrast, in the case of hypertonia of the superficial pelvic floor, and in particular of the cavernosa muscles, the possible reduction of the maximum stiffness that may occur due to a reduction of the expansion volume capacity of the corpora cavernosa roots, can benefit from appropriate therapy with electromyographic biofeedback associated with functional electrical stimulation (1).

\section{Premature Ejaculation}

Clinical trials were conducted in which the EP was treated with different rehabilitation therapy of the pelvic floor $(3,29)$. Pear $\&$ G Nicastro A (1996) published that $61 \%$ of their patients with $\mathrm{PE}$, reported greater ability to control 
the ejaculatory reflex after 15-20 sessions of rehabilitation therapy of the pelvic floor (29). The rehabilitation protocol adopted by these authors included the pelvic-perineal physiotherapy, electrical stimulation and intra-anal electromyographic biofeedback with anal probe (29).

Actual mechanism that would improve the control over the ejaculatory reflex is not clearly evident. A better management of the pelvic floor may increases awareness of this poorly corticalized area, thus improving its sense of control.

According to authoritative authors, during sexual activity, pleasure felt is amplified in both partners, from genital answers provided by the levator contraction (30). In particular, in women, the contractions would be supported by a production of myotonic $\mathrm{m}$. Pubo-coccygeal contraction that discharge during orgasm (31).

The proposed mechanism for which the active control of the musculature of the pelvic floor may delay its beginning, can be related to the inhibition of the reflex ejaculation by means of an intentional relaxation of the muscles bulbocavernosus and ischiocavernosus during arousal (1). This is a clever technique that can be learned well by using biofeedback.

\section{Sexual problems post-surgery}

Another potential of pelvic floor rehabilitation therapy, is the treatment of some cases of erectile dysfunction (ED) and/or urinary incontinence (UI) that may occur, sometimes as a complication of surgery for radical prostatectomy. Voiding and sexual iatrogenic post-surgical dysfunction, may also occur in other types of pelvic-perineal surgery in both men and women. The exact incidence of sexual dysfunction resulting from surgical pelvic-perineal procedures is not exactly known. There are, in fact, few randomized controlled trials comparing different surgical techniques in voiding function of relapses and, above all, the sexual ones (32). It is true that the UI and ED secondary to prostatectomy and pelvic-perineal surgery, can benefit from rehabilitation therapy of the pelvic floor muscles and, in particular, the use of electromyographic Biofeedbeck $(33,34)$. These results would seem to provide additional capabilities to physical therapy rehabilitation. This provided that it is left over a sufficient amount of muscle tissue on which to operate with such therapeutic methods.

\section{Chronic pelvic pain}

The male chronic pelvic pain syndrome (CPPS) is frequently associated with sexual dysfunction. These, when present, can be expressed in various manifestation: DE, EP, painful erection or, finally, painful orgasm. In a study conducted on 66 patients with turkish CPPS, EP had 51 (77.3\%), and 10 (15.2\%) had EP associated with ED (35). Chronic prostatitis-chronic pelvic pain syndrome (CPCPPS), has traditionally been associated with infectious causes or inflammation of the accessory glands, especially the prostate. In many cases, however, is difficult to document the inflammation pathogenesis. Many causes can simulate CP-CPPS symptoms such as musculoskeletal pain, dysfunction of the pelvic floor muscles, myofascial syndrome or functional somatic syndromes (36). A study of DC Hetrick (2006) comparing the pelvic floor muscles electromyography of patients with chronic pelvic pain with those of normal subjects, reported that men with pelvic pain show a tapered unstable pelvic muscles than those of normal controls (37). The same study concluded that the electromyography of the pelvic floor muscles, can be a valuable screening tool to identify patients with CP-CPPS who may benefit from a treatment aimed at correcting the dysfunction of the pelvic floor muscles, if this was present (37) as evidence of this, it was verified that the treatment of pelvic floor muscles with electromyographic biofeedback is an effective therapeutic method when applied to men with CP-CPPS $(38,39)$.

\section{Conclusions}

Sexual dysfunction is usually given by more than one factor, and certain components, biological, psychosocial and relational may contribute to dysfunction in men and women. Among the many factors involved in sexual dysfunction, pelvic floor seems to have an important role. In conclusion, the treatment of male chronic pelvic pain should also considers physiotherapy techniques that include physical therapy, therapeutic exercises, electrical stimulation and BFB. In this review of the literature on the effectiveness of each technique in the treatment of sexual dysfunction in men and women is revealed that treating pelvic dysfunction may improve sexual health and quality of life. Further randomized controlled trials are needed to validate the success of physical therapy.

\section{References}

1. Rosenbaum TY. Pelvic floor involvement in male and female sexual dysfunction and the role of pelvic floor rehabilitation in treatment: a literature review. J Sex Med. 2007; 4:4-13.

2. Dorey G, Speakman M, Feneley R, et al. Randomised controlled trial of pelvic floor muscle exercises and manometric biofeedback for erectile dysfunction. British Br J Gen Pract. 2004; 54:819-25.

3. Piediferro G, Colpi EM, Castiglioni F, Scroppo FI. Premature ejaculation. 3. Therapy. Arch Ital Urol Androl. 2004; 76:192-8.

4. Zermann DH, Ishigooka M, Doggweiler R, Schmidt RA. Chronic prostatitis: a myofascial pain syndrome Infect Urol. 1999; 12:84-92.

5. Messelink B, Benson T, Berghmans B, et al. Standardization of terminology of pelvic floor muscle function and dysfunction. Report from the pelvic floor clinical assessment group of the International Continence Society. Neurourol Urodyn. 2005; 24:374- 80.

6. G. Dorey. Conservative treatment of Male Urinary Incontinence ad Erectile Dysfunction. Whurr Publishers Ed. London and Philadelphia. 2001; 11-20.

7. Gosling JA, Dixon JS, Critchley HOD, Thompson SA. A comparative study of the human external sphincter and periurethral levator ani muscle. British Journal of Urology. 1981; 53:35-41.

8. DeLancey L. Functional anatomy of the pelvic floor and urinary continence mechanism. In B. Schüssler, J. Laycock, P Norton and S. Stanton (eds). Pelvic Floor Re-education. Principles and Practice (3nd edn). Springer-Verlag. London, 2000; 9-23.

9. Wespes E, Nogueira MC, Herbaut AG, et al. Role of the bulbocavernous muscles on the mechanism of human erection. European Urology 1990; 18:45-48. 
10. Zermann DH, Ishigooka $\mathrm{M}$, Wunderlich $\mathrm{H}$, et al. A study of pelvic floor function pre- and postradical prostatectomy using clinical neurourological investigations, urodynamics and electromyography. Eur Urol. 2000; 37:72-8.

11. Dorey G. Conservative treatment of erectile dysfunction. 3: Literature review. Br J Nurs. 2000; 9:859-63.

12. Di Benedetto P. Valutazione Neuro-Fisiatrica Perineale. In: Di Benedetto P (ed). Riabilitazione uro-ginecologica ( $\left.2^{\text {nd }} e d n\right)$. Torino, Minerva Medica. 2004; 99-103.

13. Di Benedetto P. Il bilancio muscolare perineale. In: Di Benedetto P (ed.). Riabilitazione Uro-ginecologica. Torino, Minerva Medica. 1995; 63-70.

14. Sayer T, Smith T. Pelvic floor biopsy In: B. Schüssler, J. Laycock, PA. Norton, SL. Stanton (eds.) Pelvic floor Re-education (3nd edn). Springer-Verlag. London, 2000; 98-101.

15. Gilpin SA, Gosling JA, Smith ARB, Warrell DW. The pathogenesis of genitourinary prolapse and stress incontinence of urine. A histological and histochemical study. Br J Obstet Gynaecol. 1989; 96:15-23

16. Debus-Thiede G. Magnetic resonance imaging (MRI) of pelvic floor. In B. Schüssler, J. Laycock, P Norton and S. Stanton (eds). Pelvic Floor Re-education. Principles and Practice. (3nd edn). London, Springer-Verlag. 2000; 78-82.

17. Van Kampen M, De Weerdt W, Claes H, et al. Treatment of erectile dysfunction by perineal exercise, electromyographic biofeedback, and electrical stimulation. Phys Ther. 2003; 83:536-43.

18. Strasser H, Steinlechner M, Bartsch G. Morphometric analysis of the rhabdosphincter of the male urethra. J Urol. 1997; 157(Suppl 4):177-180.

19. Wang C, Swerdloff RS, Iranmanesh A, et al. Transdermal testosterone gel improves sexual function, mood, muscle strength, and body composition parameters in hypogonadal men. J Clin Endocrinol Metab. 2000; 85:2839-2853.

20. O'Donnel PD, Doyle R. Biofeedback therapy technique for treatment of urinary incontinence. Urology. 1991; 37:432-436.

21. Laycock J. Clinical evaluation of the pelvic floor. In B. Schüssler, J. Laycock, P Norton and S. Stanton (eds). Pelvic Floor Re-education. Principles and Practice ( $\left.3^{\text {nd }} e d n\right)$. Springer-Verlag. London, 2000; 42-48

22. Wilmore J, Costill D. Physiology of sport and exercise. ( $2^{\text {nd }}$ edn). Human Kinetics, Champaign; Illinois, 1999.

23. Mansoor A, Jacquetin B, Ohana M. Evaluation des facteurs de l'incontinence urinaire féminine et indications thérapeutiques. Ann. Urol. 1993; 27:292-305

24. Vodusek D. Electrophysiology. In: B. Schüssler, J. Laycock, P Norton and S. Stanton (eds). Pelvic Floor Re-education. Principles and Practice (3nd edn). Springer-Verlag. London, 2000: 83-97.

25. Rosenbaum TY. Physiotherapy treatment of sexual pain disorders. J Sex Marital Ther. 2005; 31:329-340.

26. P. Abrams, S. Khouri, A. Wein. Incontinence. $1^{\text {st }}$ International Consultation on Incontinence. Paris:Health Publication Limited, 1999.

27. Cornel EB, Van Haarst EP, Schaarsberg RW, Geels J. The effect of biofeedback physical therapy in men with chronic pelvic pain syndrome type III. Eur Urol. 2005; 47:607-611.

28. Claes H, Baert L. Pelvic floor exercise versus surgery in the treatment of impotence. Br J Urol.1993; 71:52-7.
29. La Pera G, Nicastro A. A new treatment for premature ejaculation: The rehabilitation of the pelvic floor. J Sex Marital Ther. 1996; 22:22-6.

30. Shafik A. The role of the levator ani muscle in evacuation, sexual performance, and pelvic floor disorders. Int Urogynecol J Pelvic Floor Dysfunct. 2000; 11:361-76.

31. Komisaruk BR, Whipple B. Physiological and percentual correlates of orgasm produced by genital or non-genital stimulation. In: P. Kothari (Ed) The Proceedings of the First International Conference on Orgasm. Parthenon Press, England, 1991.

32. Achtari C, Dwyer P. Sexual function and pelvic floor disorders. Best Prac Res Clin Obstet Gynaecol. 2005; 19:993-1008.

33. Lewis RW, Fugl-Meyer KS, Bosch R, et al. Epidemiology/risk factors of sexual dysfunction. J Sex Med. 2004; 1:35-39.

34. Lue TF, Giuliano F, Montorsi F, et al. Summary of the recommendations on sexual dysfunctions in men. J Sex Med. 2004; 1:6-23.

35. Gonen M, Kalkan M, Cenker A, Ozkardes H. Prevalence of premature ejaculation in Turkish men with chronic pelvic pain syndrome. J Androl. 2005; 26:601-3.

36. Potts JM. Chronic pelvic pain syndrome: A non prostatocentric perspective. World J Urol. 2003; 21:54-6. Epub 2003. Review.

37. Hetrick DC, Glazer H, Liu YW, et al. Pelvic floor electromyography in men with chronic pelvic pain syndrome: a case control study. Neurourol Urodyn. 2006; 25:46-9.

38. Cornel EB, van Haarst EP, Schaarsberg RW, Geels J. The effect of biofeedback physical therapy in men with chronic pelvic pain syndrome type III. Eur Urol. 2005; 47:607-11.

39. YeZQ, Cai D, Du Lan RZGH, et al. Biofeedback therapy for chronic pelvic pain syndrome. Asian J Androl. 2003; 5:155-8.

\section{Correspondence}

Ferdinando Fusco, MD (Corresponding Author)

Azienda Ospedaliera Universitaria Federico II di Napoli ferdinando-fusco@libero.it

Antonella Pischedda, MD

Andrea Curreli, MD

Furio Pirozzi Farina, MD

pirozzi@uniss.it

Azienda Ospedaliera Universitaria

Viale S. Pietro 43 - 07100 Sassari, Italy

Giovanni Grimaldi, MD

Azienda Ospedaliera Universitaria Federico II - Napoli, Italy 